\title{
Detection of HPV subtypes by mass spectrometry in FFPE tissue specimens: a reliable tool for routine diagnostics
}

\author{
Mark Kriegsmann, ${ }^{1}$ Petra Wandernoth, ${ }^{2}$ Katharina Lisenko, ${ }^{3}$ Rita Casadonte, ${ }^{4}$ \\ Rémi Longuespée, ${ }^{4}$ Norbert Arens, ${ }^{2}$ Jörg Kriegsmann ${ }^{2,5}$
}

\begin{abstract}
Institute of Pathology, University of Heidelberg, Heidelberg, Germany Institute of Molecular Pathology, Trier, Germany ${ }^{3}$ Department of Internal Medicine $V$, University of Heidelberg, Heidelberg, Germany

${ }^{4}$ Proteopath GmbH, Trier, Germany

${ }^{5} \mathrm{MVZ}$ for Histology, Cytology and Molecular Diagnostics, Trier, Germany
\end{abstract}

Correspondence to Mark Kriegsmann, Institute of Pathology, University Hospital Heidelberg, Im Neuenheimer Feld 224, Heidelberg 69120, Germany; mark.kriegsmann@ med.uni-heidelberg.de

MK and PW contributed equally to this work.

Received 8 July 2016 Revised 21 September 2016 Accepted 23 September 2016 Published Online First 8 October 2016

CrossMark

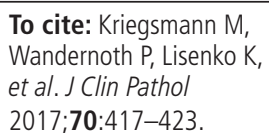

\section{ABSTRACT}

Aims Human papilloma virus (HPV) infection is a causative agent for approximately $5 \%$ of all new cancer cases in humans. The virus is detected in cervical, anal, vaginal, penile, vulvar and head and neck cancers and has prognostic implications. Thus, test systems are required to detect high-risk but also low-risk HPV subtypes with high specificity and sensitivity in a timeeffective and cost-effective manner. In the present study we developed a new mass spectrometry (MS)-based test system for the detection of HPV infections in formalinfixed paraffin-embedded (FFPE) tissue samples.

Methods A high-throughput matrix-assisted laser desorption ionisation time of flight MS-based assay was applied to genotype 19 HPV types in FFPE tissue specimens $(n=46)$. The results from the MS assay were compared with the results obtained from two hybridisation-based test systems: the HPV 3.5 LCD-array kit and the EuroArrayHPV system.

Results In 36 out of 46 (78\%) tissue samples, a HPV infection could be detected by the MS-based HPV assay. In 16 samples (44\%) only one and in 20 samples (56\%) two to six HPV subtypes were identified. The overall agreement of all three assays was almost perfect (Cohen's $k$ value: 0.83 ).

Conclusions The MS-based assay is highly sensitive, reliable as well as cost-effective and represents a suitable technology for the detection of HPV infections in FFPE tissue material.

\section{INTRODUCTION}

Oncogenic human papilloma virus (HPV) strains are detected in almost all cervical cancers $(100 \%)$, the majority of anal cancers (88\%), vaginal cancers (70\%), penile cancers $(50 \%)$ and vulvar cancers (43\%) and in a subset of head and neck cancer (up to $\sim 50 \%){ }^{1}$ In 2008 , approximately 610000 of the 12.7 million new cancer cases were attributable to HPV with worldwide 570000 and 39000 new cancer cases diagnosed among women and men, respectively. ${ }^{2}$

Despite the fact that cytological screening has been available since more than five decades, cervical cancer remains the fourth most common cancer in women. ${ }^{3}$ Among others, the infection with high-risk HPV subtypes is considered the most important risk factor. ${ }^{4}$ Persistent infection of squamous epithelia with one of about 15 genotypes of HPV leads over different steps from low-grade to high-grade squamous intraepithelial lesions/cervical intraepithelial neoplasia 1-3 and subsequently to invasive carcinoma. Apparently, each genotype of
HPV acts as an independent infection, with variable carcinogenic risk linked to evolutionary species. ${ }^{4}$ Our understanding of HPV as carcinogenic agent has led to improved clinical management strategies such as the implementation of HPV vaccination and the development of new molecular screening assays in cervical cancer. ${ }^{5-7}$

Head and neck cancer with squamous cell histology $(\sim 90 \%)$ is the seventh most common cancer worldwide and is located in the oral cavity, the oropharynx, the nasal cavity, paranasal sinuses, the nasopharynx, the hypopharynx and the larynx. The aetiology is based on one of two primary carcinogenic routes: (a) chemical carcinogenesis through exposure to tobacco and alcohol abuse and (b) highrisk HPV. While the incidence of head and neck cancer attributable to chemical agents is decreasing, the incidence of HPV-related oropharyngeal cancer is currently increasing, particularly in young people. ${ }^{1}$ Determination of HPV-positive and HPV-negative head and neck cancer has prognostic implications, as HPV-positive head and neck cancer has a favourable prognosis as compared with HPV-negative head and neck cancer. ${ }^{8}$ Interestingly, oropharyngeal cancer incidence is expected to surpass cervical cancer incidence by 2020 in the USA. ${ }^{19}$

Besides the skin, the genital tract and the head and neck region, HPV has been detected in various organs including lung, prostate, breast, bladder and stomach. ${ }^{10}$ Despite the fact that some interesting associations have been proclaimed, for all of these organs more studies are necessary before HPV testing can be recommended.

However, it is foreseeable that pathologists and biologists will be faced with a rising number of HPV tests in the near future. Test systems are required to detect high-risk but also low-risk HPV subtypes with high specificity and sensitivity in a time-effective and cost-effective manner. Recently, we have shown that mass spectrometry (MS)-based test systems can be incorporated in routine diagnostics to provide fast and reliable results for clinicians and patients. In the present study, we developed a customised MS-based assay to test for HPV infections on formalin-fixed paraffin-embedded (FFPE) tissue specimens and compared the results with two hybridisation-based test systems that are used in routine diagnostics: the LCD-array kit and the EuroArrayHPV system.

\section{METHODS AND MATERIALS \\ Patients and sample handling}

A retrospective single-centre analysis of patients with positive HPV status was performed. Samples 
from 2012 to 2016 were retrieved from the archives of the 'Molekularpathologie Trier'. A total of 46 FFPE tissue samples were collected for analysis. Informed consent for sample testing was given by all patients (male: $n=25$; female: $n=21$ ). Tissue was derived from perianal $(n=21)$, cervix uteri $(n=8)$, vulva $(n=9)$, oropharynx $(n=4)$, vagina $(n=2)$ and skin of testis $(n=2)$. Samples were tested for a new custom-designed HPV assay for routine MS analysis (Agena Bioscience, Hamburg, Germany) and compared with two hybridisation assays: the LCD-array kit (Chipron GmbH, Berlin, Germany) and the EuroArrayHPV system (Euroimmun, Luebeck, Germany).

\section{DNA extraction from FFPE samples}

H\&E-stained slides from FFPE tissue samples were reviewed by a pathologist and squamous epithelium was macroscopically dissected in order to extract DNA as described previously. ${ }^{11}$ In brief, dissected tissues were transferred to $200 \mu \mathrm{L}$ lysis buffer (ATL Tissue lysis buffer; catalogue no. 939011, Qiagen, Hilden, Germany) and treated for $1 \mathrm{~h}$ with $20 \mu \mathrm{L}$ Proteinase $\mathrm{K}(20 \mathrm{mg} / \mathrm{mL}$; catalogue no. 19133, Qiagen, Hilden, Germany) at $56^{\circ} \mathrm{C}$. After proteinase $\mathrm{K}$ inactivation $\left(1 \mathrm{~h}, 90^{\circ} \mathrm{C}\right)$ and centrifugation $(1 \mathrm{~min}, 300 \mathrm{~g})$, DNA was semiautomatically isolated using a QiaSymphony and the DNA mini Kit (catalogue no. 937236, Qiagen, Hilden, Germany). DNA concentration was determined by OD $260 \mathrm{~nm}$ with the NanoDrop spectrophotometry (Peqlab, Erlangen, Germany).

\section{MS-based assay}

HPV detection was carried out using an iPlex matrix-assisted laser desorption ionisation time of flight (MALDI-TOF) mass spectrometer (Agena Bioscience, Hamburg, Germany). The customised panel included the detection of 19 HPV subtypes (table 1) combined in one multiplex assay. The assay included all subtypes that are International Agency for Research on Cancer (IARC) group 1 (carcinogenic) and 2a (probably carcinogenic), thus all subtypes of HPV that are considered high risk. Moreover, relevant HPV subtypes from IARC group $2 \mathrm{~b}$ (possibly carcinogenic) were also included. The experiments were performed with the complete iPlex Pro Reagent Set (catalogue no. 10160, Agena Bioscience) and the manufacturer's standard protocol (Agena Bioscience). The workflow is schematically depicted in figure 1 . High-quality DNA is required and expected to be diluted to $5-10 \mathrm{ng} / \mu \mathrm{L}$. Virus DNA was amplified by using the supplied HPV PCR primers in one 96-well plate in a final reaction volume of $5 \mu \mathrm{L}$. The HPV PCR primers are premixed for the assay panel (catalogue no. 17902, Agena Bioscience) and contain target type-specific base pairs in the E6/E7 region of the HPV genome for 19 HPV subtypes. Amplification occurs by the following PCR conditions: First step 1 cycle $95^{\circ} \mathrm{C} 2 \mathrm{~min}$; second step 45 cycles $95^{\circ} \mathrm{C} 30 \mathrm{~s}, 56^{\circ} \mathrm{C} 30 \mathrm{~s}, 72^{\circ}$
C; third step 1 cycle $72^{\circ} \mathrm{C} 5 \mathrm{~min}$ and finally $4^{\circ} \mathrm{C} \infty$. Resulting amplicon lengths of the different HPV subtypes and the internal control glyceraldehyde-3-phosphate dehydrogenase (GAPDH) are listed in table 2. To remove surplus nucleotides each PCR reaction was treated with a shrimp-alkaline-phosphatase (SAP) mix and incubated on a thermocycler with the following programme: $37^{\circ} \mathrm{C}$ $40 \mathrm{~min}, 85^{\circ} \mathrm{C} 5 \mathrm{~min}$ and $4^{\circ} \mathrm{C} \infty$. The extension reaction is identified by using mass-modified terminator nucleotides, which elongate the amplified DNA strands at the nucleotide position of interest. Dependent on the existent virus, DNA hybridises to a different mass-modified nucleotide, which is crucial for the TOF detection by MALDI. Therefore, $2 \mu \mathrm{L}$ of the extend reaction mix that includes HPV extend primers (catalogue no. 17902, Agena Bioscience) is added to the SAP-treated PCR product and run the following cycler programme: First step 1 cycle $94^{\circ} \mathrm{C} 30 \mathrm{~s}$, second step 40 cycles $94^{\circ} \mathrm{C} 5 \mathrm{~s}, 5$ cycles $\left(52^{\circ} \mathrm{C} 5 \mathrm{~s}, 80^{\circ} \mathrm{C} 5\right.$ s), third step 1 cycle $72^{\circ} \mathrm{C} 3 \mathrm{~min}$ and finally $4^{\circ} \mathrm{Co}$. After the samples were mixed with nanopure water $(>18 \mathrm{~m} \Omega)$ and desalted with $15 \mathrm{mg}$ clean resin on a rotate plate for $20 \mathrm{~min}$ at room temperature, $10-20 \mu \mathrm{L}$ was spotted on a matrix-precoated Spectro-CHIP (Nanodispenser RS1000, Agena Bioscience). The distinct masses were determined and analysed by the MS instrument (Analyser 4, Agena Bioscience). The final report was automated on the MassArrayTyperAnalyser software V.3.3 (Agena Bioscience). The report included a spectrum view and a list of all positive HPV types. An internal GAPDH control to pass or fail DNA quality was included. Samples that did not contain any HPV subtypes have been automatically listed as negative.

\section{HPV LCD-array kit}

The HPV 3.5 LCD-array kit is a chip detection system to identify $40 \mathrm{HPV}$ subtypes (table 1). PCR, hybridisation and detection were performed according to the manufacturer's protocol (catalogue no. V-100-12, Chipron, Berlin, Germany). Ten to $50 \mathrm{ng}$ of isolated DNA were used for two separate biotinlabelled PCR amplifications. Biotin-labelled products were pooled and hybridised in the next step with immobilised specific capture probes on the LCD chip. Visualisation of binding was displayed by a streptavidin-based enzyme-substrate illumination. Fully automated read-out and report generation was done with the SlideReader software V.7.01 (Chipron).

\section{EuroArrayHPV system}

The EuroArray HPV system (EuroImmun) is a chip detection system to identify $30 \mathrm{HPV}$ subtypes (table 1). The test system is based on the detection of the viral oncogenes E6/E7. PCR, hybridisation and detection were performed according to the manufacturer's protocol. First, a PCR reaction was performed

Table 1 HPV subtypes included in the assays

\begin{tabular}{|c|c|c|c|}
\hline Risk classification & $\begin{array}{l}\text { HPV subtypes detected by the mass } \\
\text { spectrometry-based assay }\end{array}$ & $\begin{array}{l}\text { HPV subtypes detected by the HPV } \\
\text { LCD-array }\end{array}$ & $\begin{array}{l}\text { HPV subtypes detected by the } \\
\text { EuroArrayHPV }\end{array}$ \\
\hline IARC group 1 (carcinogenic) & $16,18,31,33,35,39,45,51,52,56,58,59$ & $\begin{array}{l}16,18,31,33,35,39,45,51,52,56 \\
58,59\end{array}$ & $\begin{array}{l}16,18,31,33,35,39,45,51,52,56, \\
58,59\end{array}$ \\
\hline $\begin{array}{l}\text { IARC group } 2 \mathrm{~A} \text { (probably } \\
\text { carcinogenic) }\end{array}$ & 68 & 68 & 68 \\
\hline $\begin{array}{l}\text { IARC group 2B (possibly } \\
\text { carcinogenic) }\end{array}$ & $53,66,67,73$ & $\begin{array}{l}5,8,26,30,34,53,66,67,69,70, \\
73,82,85,97\end{array}$ & $26,53,66,70,73,82$ \\
\hline IARC group 3 (not classifiable) & 6,11 & 6,11 & 6,11 \\
\hline No group & & $\begin{array}{l}42,44,54,61,62,72,81,83,84,90 \text {, } \\
91\end{array}$ & $40,42,43,44,54,61,72,81,89$ \\
\hline
\end{tabular}

HPV, human papilloma virus; IARC, International Agency for Research on Cancer. 


\section{HPV subtyping workflow by MALDI TOF analysis}

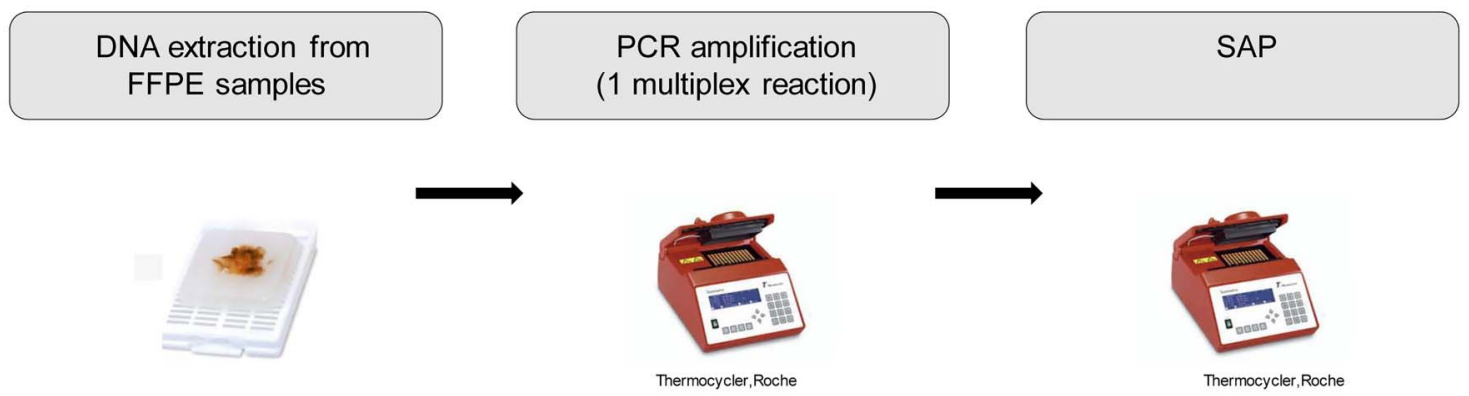

day 1
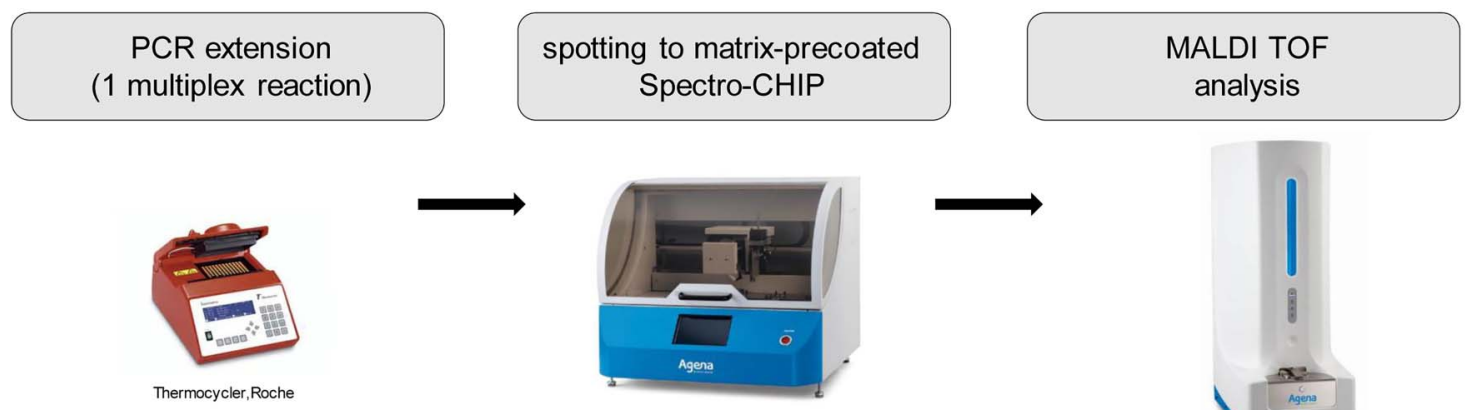

Thermocycler, Roche
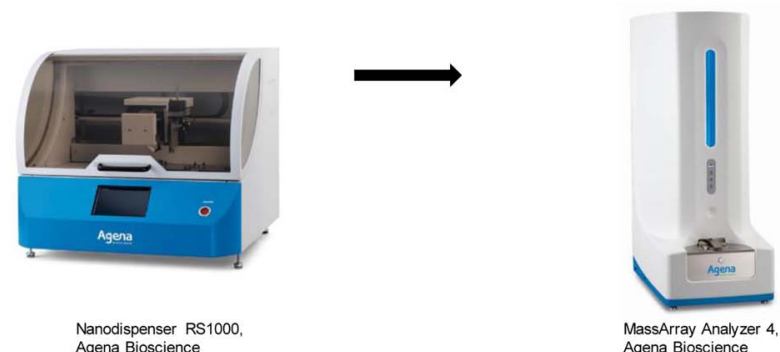

Nanodispenser RS1000 Agena Bioscience

Figure 1 A schematic workflow of a HPV analysis by mass spectrometry. First, DNA is extracted from FFPE tissue sections. Second, a PCR amplification is performed. Third, surplus nucleotides are removed by treatment with SAP. Fourth, a PCR extension reaction is carried out. Fifth, the solution is spotted to a matrix-precoated CHIP that is subsequently analysed by the MALDI-TOF instrument. FFPE, formalin-fixed paraffin-embedded; HPV, human papilloma virus; MALDI-TOF, matrix-assisted laser desorption ionisation time of flight; SAP, shrimp-alkaline-phosphatase.

Table 2 Amplicon lengths of the different HPV subtypes and the internal control GAPDH

\begin{tabular}{lc}
\hline SNP ID & Amplicon length (bp) \\
\hline HPV16 & 141 \\
HPV18 & 101 \\
HPV31 & 111 \\
HPV33 & 112 \\
HPV35 & 126 \\
HPV39 & 131 \\
HPV45 & 101 \\
HPV51 & 112 \\
HPV52 & 100 \\
HPV56 & 103 \\
HPV58 & 81 \\
HPV59 & 121 \\
HPV68 & 103 \\
HPV53 & 122 \\
HPV66 & 88 \\
HPV67 & 99 \\
HPV73 & 124 \\
HPV6 & 96 \\
HPV11 & 100 \\
GAPDH & 177 \\
\hline GAPDH, glyceraldehyde-3-phosphate-dehydrogenase; HPV, human papilloma virus; \\
SNP, single nucleotide polymorphism. \\
\end{tabular}

using a multiplex primer system and at the same time labelled with a fluorescent dye. Second, the products were detected using an oligonucleotide microarray. The specific binding (hybridisation) of a fluorescently labelled PCR product to its corresponding oligonucleotide probe was identified using the EUROIMMUN Microarray Scanner (EuroImmun). The EUROArrayScan software (EuroImmun) evaluated all spot signals automatically and deducts the test result.

\section{Statistics}

Data analysis was performed using $\mathrm{R}$ statistical software (V.3.2.2.), RStudio (V.0.98.507) and Excel 2010 (Microsoft, Redmond, Washington, USA). Cohen's statistic was performed in $\mathrm{R}$ using the irr package (V.0.84) to test for agreement. $\kappa$ values from 0.00 to 0.20 were considered slight agreement, from 0.21 to 0.40 fair agreement, from 0.41 to 0.60 moderate agreement, from 0.61 to 0.80 substantial agreement and from 0.81 to 1.00 almost perfect agreement. Almost perfect agreement of $\kappa$ values of all three tests was taken as an indication of high sensitivity of the test for the respective HPV subtype. Negative $\kappa$ values indicated active disagreement.

\section{RESULTS}

Frequencies of detected HPV infections by the MS-based assay

In 36 out of $46(78 \%)$ tissue samples a HPV infection could be detected by our customised MS HPV assay. In 16 samples (44\%) 

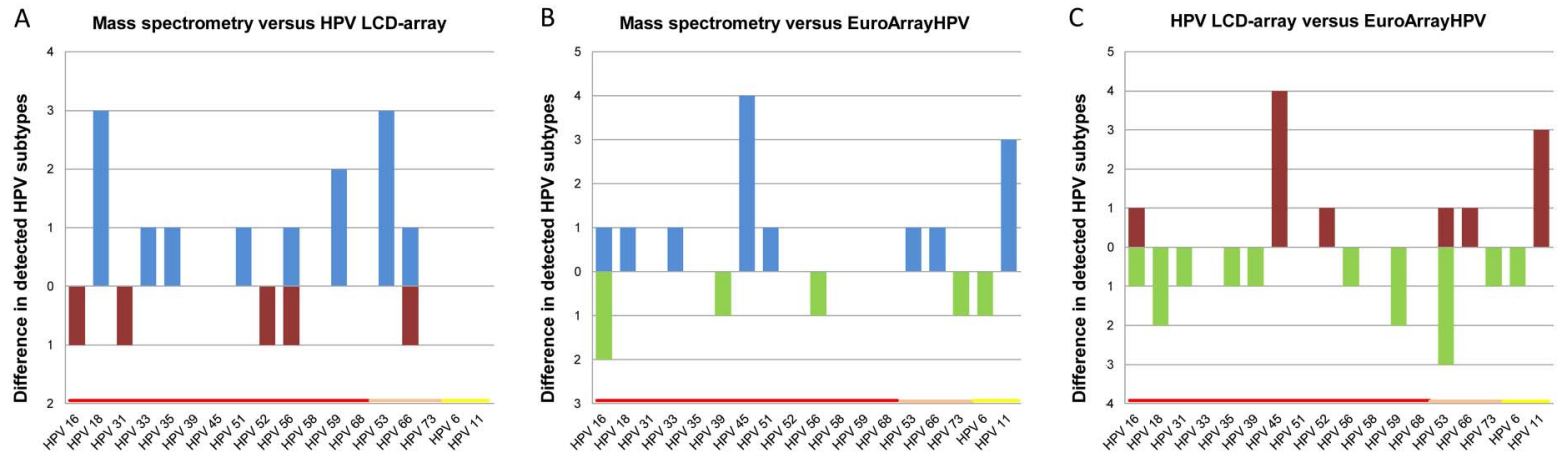

Figure 2 Comparison of differences in detected HPV subtypes. Only HPV subtypes that are detected by all three methods are depicted. Mass spectrometry (blue) detected more HPV infections as compared with the LCD-array (red) (A) and the EuroArrayHPV (green) (B). The LCD-array (red) detected less HPV infections than the EuroArrayHPV test (green) (C). High-risk HPV subtypes are highlighted in red, low-risk HPV subtypes in yellow. HPV, human papilloma virus.

only one HPV subtype could be identified, and in the remaining 20 samples $(56 \%)$ two or more HPV subtypes were detected. Tissue samples derived from the following locations showed HPV positivity: perianal (18/21), vulva (6/9), cervix (7/8), oropharynx (4/4), vagina (2/2) and skin of testis (1/2). Forty (55\%), $0(0 \%), 13(18 \%)$ and $20(27 \%)$ HPV subtypes were identified in IARC groups $1,2 \mathrm{~A}, 2 \mathrm{~B}$ and 3 , respectively. The most frequently detected HPV subtypes from group 1 were $16(23 \%)$, $18(15 \%)$ and $45(13 \%)$, from group 2B were $53(31 \%), 73$ $(31 \%)$ and $66(23 \%)$ and from group 3 were $6(55 \%)$ and 11 (45\%). GAPDH controls were positive in all cases tested.

\section{Frequencies of detected HPV infections by the HPV LCD-array kit}

In 38 out of $46(83 \%)$ tissue samples a HPV infection could be detected by the HPV LCD-array kit. In 18 samples (47\%) only one HPV subtype could be identified, and in the remaining 20 samples $(53 \%)$ two or more HPV subtypes were detected. Thirty-two (72\%), 0 (0\%), 11 (14\%) and 20 (26\%) HPV subtypes were identified in IARC groups $1,2 \mathrm{~A}, 2 \mathrm{~B}$ and 3 , respectively. Moreover, $13(17 \%)$ HPV subtypes that are not yet classified according to IARC were detected.

The most frequently detected HPV subtypes from group 1 were $16(31 \%), 45(16 \%)$ and 18, 31, 56 (9\% each), from group 2B were 73 (36\%), 66 (18\%) and 67, 82 (18\% each) and from group 3 were $6(55 \%)$ and $11(45 \%)$. Among the HPV subtypes that are not yet classified, the most frequently identified were 91 (38\%), 42 (23\%) and 44, 72, 81, 84 and 90 (8\% each).

\section{Frequencies of detected HPV infections by the EuroArrayHPV system}

In 37 out of $46(80 \%)$ tissue samples a HPV infection could be detected by the EuroArrayHPV system. In 17 samples (46\%) only one HPV subtype could be identified, and in the remaining 20 samples $(54 \%)$ two or more HPV subtypes were detected. Thirty-seven (46\%), 0 (0\%), 12 (15\%) and 19 (24\%) HPV subtypes were identified in IARC groups $1,2 \mathrm{~A}, 2 \mathrm{~B}$ and 3 , respectively. Additionally, $12(15 \%) \mathrm{HPV}$ subtypes that are not yet classified according to the IARC were detected.

The most frequently detected HPV subtypes from group 1 were $16(30 \%), 18(14 \%), 31,56$ (11\% each), from group 2B were $73(42 \%), 53(33 \%)$ and 66, 82 (17\% each), and from group 3 were $6(63 \%)$ and 11 (37\%). Among the HPV subtypes that are not yet classified the most frequently identified were 42 (33\%), 44 (17\%) and 40, 43, 54, 61, 72, 89 (8\% each).

\section{Comparison between the three test systems}

When only HPV subtypes were taken into account that can be detected by all three test systems, the MS-based assay, the LCD-array kit and the EuroArrayHPV, the overall agreement was almost perfect ( $\mathrm{k}$ value: 0.828 ).

The MS-based assay detected nine more and four less HPV infections in IARC group 1 and four more and one less HPV infections in IARC group $2 \mathrm{~B}$ as compared with the LCD-array kit (figure 2A). Agreement of both tests was mainly substantial or almost perfect (overall k value: 0.852). Exceptions were HPV 53 (fair agreement, $\mathrm{k}$ value: 0.378) and HPV 59 (moderate agreement, $\mathrm{k}$ value: 0.483 ) where the MS-based assay detected more HPV infections. Interestingly, the $\mathrm{k}$ value of these HPV subtypes was very high between the MS-based assay and the EuroArrayHPV test system.

As compared with the EuroArrayHPV system, eight HPV subtypes more and four less HPV infections from IARC group 1, two more and one less HPV infections from IARC group $2 \mathrm{~B}$ and three more and one less HPV infections from IARC group 3 were detected by the MS-based assay (figure 2B). Again, the agreement was mainly substantial or almost perfect (overall $\mathrm{k}$ value: 0.847 ). HPV 45 showed only fair agreement ( $\mathrm{k}$ value: 0.308). Of note, HPV infections with this HPV subtype had been detected with perfect agreement with the MS-based assay and the HPV LCD-array kit (k value: 1.00$)$.

Compared with the EuroArrayHPV system, the HPV LCD-array kit identified six more and nine less HPV infections in IARC group 1, two more and four less HPV infections from IARC group $2 \mathrm{~B}$ and three more and one HPV infections less than in IARC group 3 (figure 2C). Agreement between the HPV LCD-array kit and the EuroArrayHPV test system was mainly substantial or almost perfect (overall $\mathrm{k}$ value: 0.78 ). Exceptions were HPV 53 (poor agreement, k value: -0.034), HPV 45 (fair agreement, k value: 0.308 ) and HPV 59 (moderate agreement, $\mathrm{k}$ value: 0.483). All HPV subtypes showed almost perfect agreement with the MS-based assay and the HPV LCD-array kit ( $\mathrm{k}$ value $>0.81$ ). A summary of all detected HPV infections and $\mathrm{k}$ values is provided in table 3. An example of a case with multiple simultaneous HPV infections is depicted in figure 3 .

\section{DISCUSSION}

In the last decade, our knowledge on HPV as a carcinogenic agent has been expanded. The use of HPV testing as part of cervical cancer prevention and the implementation of HPV vaccines has led to a decline in cervix cancer incidence and 
Table 3 Frequencies and concordances across different HPV subtypes between the three assays

\begin{tabular}{|c|c|c|c|c|c|c|c|}
\hline Parameters & $\begin{array}{l}\text { HPV } \\
\text { subtype }\end{array}$ & $\begin{array}{l}\text { MS-based } \\
\text { assay }\end{array}$ & $\begin{array}{l}\text { HPV } \\
\text { LCD-array kit }\end{array}$ & $\begin{array}{l}\text { EuroArrayHPV } \\
\text { system }\end{array}$ & $\begin{array}{l}\text { MS vs HPV } \\
\text { LCD-array } \\
\text { (k value) }\end{array}$ & $\begin{array}{l}\text { MS vs EuroArrayHPV } \\
\text { system (k value) }\end{array}$ & $\begin{array}{l}\text { HPV LCD-array kit vs } \\
\text { EuroArrayHPV system } \\
\text { (k value) }\end{array}$ \\
\hline IARC group 1, n (\%) & & $40(55)$ & $32(42)$ & $37(46)$ & & & \\
\hline \multirow[t]{12}{*}{$\mathrm{n}$ (\% of group 1 ) } & 16 & $9(23)$ & $10(31)$ & $11(30)$ & 0.934 & 0.801 & 0.872 \\
\hline & 18 & $6(15)$ & $3(9)$ & $5(14)$ & 0.635 & 0.897 & 0.728 \\
\hline & 31 & $4(10)$ & $3(9)$ & $4(11)$ & 0.846 & 1.000 & 0.846 \\
\hline & 33 & $3(8)$ & $2(6)$ & $2(5)$ & 0.789 & 0.789 & 1.000 \\
\hline & 35 & $3(8)$ & $2(6)$ & $3(8)$ & 0.789 & 1.000 & 0.789 \\
\hline & 39 & $1(3)$ & $1(3)$ & $2(5)$ & 1.000 & 0.657 & 0.657 \\
\hline & 45 & $5(13)$ & $5(16)$ & $1(3)$ & 1.000 & 0.308 & 0.308 \\
\hline & 51 & $2(5)$ & $1(3)$ & $1(3)$ & 0.657 & 0.657 & 1.000 \\
\hline & 52 & $1(3)$ & $2(6)$ & $1(3)$ & 0.657 & 1.000 & 0.657 \\
\hline & 56 & $3(8)$ & $3(9)$ & $4(11)$ & 0.643 & 0.846 & 0.846 \\
\hline & 58 & $0(0)$ & $0(0)$ & $0(0)$ & NA & NA & NA \\
\hline & 59 & $3(8)$ & $1(3)$ & $3(8)$ & 0.483 & 1.000 & 0.483 \\
\hline IARC group $2 \mathrm{~A}, \mathrm{n}(\%)$ & & $0(0)$ & $0(0)$ & $0(0)$ & & & \\
\hline$n(\%$ of group $2 A)$ & 68 & $0(0)$ & $0(0)$ & $0(0)$ & NA & NA & NA \\
\hline IARC group $2 \mathrm{~B}, \mathrm{n}(\%)$ & & $13(18)$ & $11(14)$ & $12(15)$ & & & \\
\hline \multirow[t]{14}{*}{$n(\%$ of group $2 B)$} & 5 & NA & $0(0)$ & NA & NA & NA & NA \\
\hline & 8 & NA & $0(0)$ & NA & NA & NA & NA \\
\hline & 26 & NA & $0(0)$ & $1(8)$ & NA & NA & NA \\
\hline & 30 & NA & $0(0)$ & NA & NA & NA & NA \\
\hline & 34 & NA & $0(0)$ & NA & NA & NA & NA \\
\hline & 53 & $4(31)$ & $1(9)$ & $4(33)$ & 0.378 & 0.846 & -0.034 \\
\hline & 66 & $3(23)$ & $3(27)$ & $2(17)$ & 0.643 & 0.789 & 0.789 \\
\hline & 67 & $2(15)$ & $2(18)$ & NA & 1.000 & NA & NA \\
\hline & 69 & NA & $0(0)$ & NA & NA & NA & NA \\
\hline & 70 & NA & $0(0)$ & $0(0)$ & NA & NA & NA \\
\hline & 73 & $4(31)$ & $4(36)$ & $5(42)$ & 1.000 & 0.877 & 0.877 \\
\hline & 82 & NA & $2(18)$ & $2(17)$ & NA & NA & 1.000 \\
\hline & 85 & NA & $0(0)$ & NA & NA & NA & NA \\
\hline & 97 & NA & $0(0)$ & NA & NA & NA & NA \\
\hline IARC group 3, n (\%) & & $20(27)$ & $20(26)$ & $19(24)$ & & & \\
\hline \multirow[t]{2}{*}{ n (\% of group 3) } & 6 & $11(55)$ & $11(55)$ & $12(63)$ & 1.000 & 0.942 & 0.942 \\
\hline & 11 & $9(45)$ & $9(45)$ & $7(37)$ & 1.000 & 0.763 & 0.763 \\
\hline IARC; no group, $\mathrm{n}(\%)$ & & NA & $13(17)$ & $12(15)$ & & & \\
\hline \multirow[t]{14}{*}{ n (\% of no group) } & 40 & NA & NA & $1(8)$ & NA & NA & NA \\
\hline & 42 & NA & $3(23)$ & $4(33)$ & NA & NA & 0.846 \\
\hline & 43 & NA & NA & $1(8)$ & NA & NA & NA \\
\hline & 44 & NA & $1(8)$ & $2(17)$ & NA & NA & 0.657 \\
\hline & 54 & NA & $0(0)$ & $1(8)$ & NA & NA & NA \\
\hline & 61 & NA & $0(0)$ & 1 (8) & NA & NA & NA \\
\hline & 62 & NA & $0(0)$ & NA & NA & NA & NA \\
\hline & 72 & NA & $1(8)$ & $1(8)$ & NA & NA & 1.000 \\
\hline & 81 & NA & $1(8)$ & $0(0)$ & NA & NA & NA \\
\hline & 83 & NA & $0(0)$ & NA & NA & NA & NA \\
\hline & 84 & NA & $1(8)$ & NA & NA & NA & NA \\
\hline & 89 & NA & NA & 1 (8) & NA & NA & NA \\
\hline & 90 & NA & $1(8)$ & NA & NA & NA & NA \\
\hline & 91 & NA & $5(38)$ & NA & NA & NA & NA \\
\hline
\end{tabular}

HPV, human papilloma virus; IARC, International Agency for Research on Cancer; MS, mass spectrometry.

cancer-related death. ${ }^{12}$ More recently, HPV has been detected in oropharyngeal cancer and it became clear that HPV-related head and neck cancer form a distinct entity with particular clinicopathological characteristics. Several molecular targeted strategies are under discussion to improve chemoradiation response. ${ }^{9}$ Thus, it is clear that broad-range HPV testing will enter clinical practice.
It is generally acknowledged that certain HPV infections stratify the risk for cervical cancer and that coinfection with multiple HPV subtypes is associated with an increased risk. ${ }^{4}$ This is particularly important since infection with multiple HPV subtypes may lead to treatment failure. ${ }^{13}$ In an attempt to classify the HPV subtypes according to their carcinogenic risk, the IARC established a classification system with four categories. Group 1 
Figure 3 Example of a case with multiple HPV infections detected by mass spectrometry. A mass range from 4500 to 9000 is shown. UEP and pos are highlighted in a colour-encoded way (A). A zoomed view in the mass range from 4900 to 5600 illustrates three small peaks corresponding to the remaining extension primer rests from HPV35 (left green line), GAPDH control (left blue line) and HPV18 (left pink line), as well as the amplification product peaks from HPV35 (right green line), GAPDH (right blue line) and HPV18 (right pink line) (B). GAPDH, glyceraldehyde-3-phosphate dehydrogenase; HPV, human papilloma virus; pos, amplification products; UEP, unextended primers.

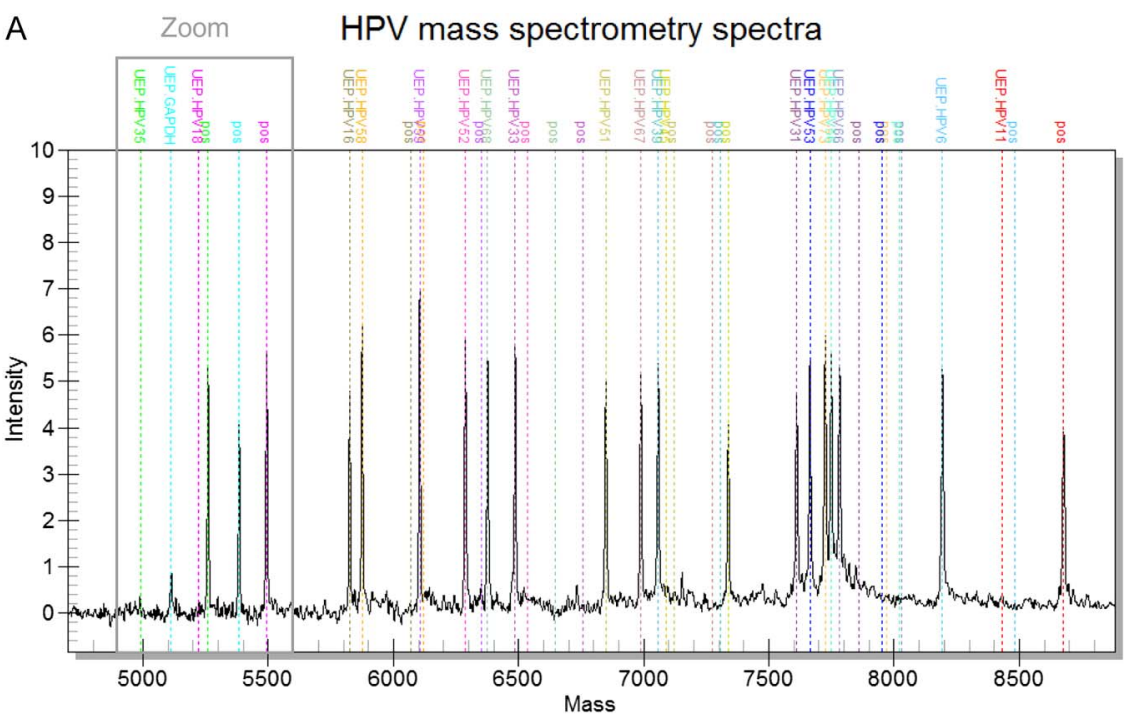

B

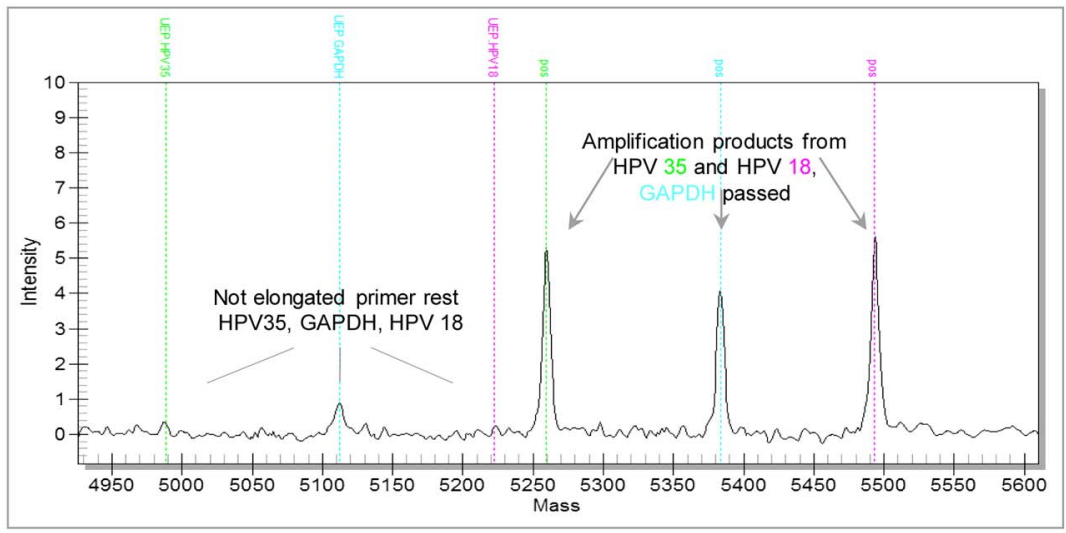

incorporates carcinogenic, group 2 probably carcinogenic, group 3 possibly carcinogenic and group 4 HPV subtypes uncertain carcinogenic risk (table 1). Thus, accurate genotyping is very important for risk assessment and monitoring of HPV-positive patients.

Therefore, test systems are required to detect high-risk but also low-risk HPV subtypes with high specificity and sensitivity in a time-effective and cost-effective manner. Although virtually all test systems to detect HPV infections cover the HPV subtypes from group 1 and group 2A, only selected HPV subtypes from group $2 \mathrm{~B}$ and group 3 are included in different test systems. ${ }^{14} 15$ Since no guidelines are available for HPV testing, it is not entirely clear which HPV subtypes from group $2 \mathrm{~B}$ and group 3 should be incorporated into clinical tests.

MS as a method to detect PCR amplification products is well established and has gained great acceptance for the identifications of genetic mutations. ${ }^{16}$ Flexible testing for additional PCR products is possible, and hands-on time is generally comparable to the chip-based assays and reduced compared with sequencing analyses. ${ }^{11}$ Recently, we have shown that MS-based test systems can be used in routine diagnostics to provide fast and reliable results for clinicians and patients. ${ }^{11}$ MS can be applied to detect HPV subtypes and large clinical trials have been conducted with MS as the detection method for HPV on cytology specimens. ${ }^{17-20}$ Both the $\mathrm{L} 1^{1921}$ and the E6/E7 ${ }^{14} 22{ }^{23}$ gene have been targeted. However, there is still a need to compare MS-based assays with hybridisationbased test systems that are currently used by many institutes of pathology. This is particularly true for FFPE tissue specimens.
Although the patient size was limited and the overall agreement between the tests was very high, our results indicate that the detection of HPV types 18, 59 and 53 particularly challenged the HPV LCD-array kit and that the detection of HPV type 45 challenged the EuroArrayHPV system.

In only a very few cases, we could not confirm positive results obtained by our MS-based assay. This is true for both group 1 and group 2A/B, HPV subtypes. Although we believe that these differences may be attributed to the higher sensitivity of our MS-based assay, it is possible that these represent false-positive results. Despite adequate safety measures to avoid contaminations as a possible source of false positives, such as the entrainment of control samples, this risk cannot be entirely eliminated. However, this risk is identical among all test systems.

With regard to certainty of the detected HPV infection, we favour the MS-based assay and the EuroArrayHPV system over the HPV LCD-array kit, since no internal controls were included in the latter. Furthermore, automated systems as MS and EuroArrayHPV are less prone to subjective interpretations errors. Of note, in the 10 patients where no HPV infection could be detected by the MS-based assay, none of the other two tests showed a positive result considering the HPV subtypes included in all tests. This suggests a very high specificity of our MS-based approach.

In the present study, we investigated FFPE tissue samples from 46 patients for HPV infections by MS and compared the results with two hybridisation assays: the LCD-array and the EuroArrayHPV system. Our customised MS-based assay reliably 
detected HPV subtypes and is therefore a good candidate for routine use. In accordance to previous studies we can confirm that MS is highly sensitive, reliable and cost-effective when applied in institutions with a high case load. ${ }^{17} 19$

\section{Take home messages}

- Mass spectrometry is applicable on formalin-fixed paraffin-embedded tissue specimens.

- Mass spectrometry-based test systems are suitable to detect high-risk and low-risk human papilloma virus subtypes.

- The performance of mass spectrometry-based test systems is non-inferior to hybridisation-based test systems.

\section{Handling editor Runjan Chetty}

Contributors MK and PW drafted the manuscript. PW and NA acquired the data. $\mathrm{MK}$ and $\mathrm{KL}$ analysed the data. MK, KL and JK interpreted the data. RC, RL, KL, NA and JK revised the manuscript for important intellectual content. All authors approved the final version of the manuscript.

Funding MK is supported by the post-doc programme of the medical faculty of the University of Heidelberg.

Competing interests None declared.

Patient consent Obtained.

Ethics approval Ethics Committee of the University of Heidelberg.

Provenance and peer review Not commissioned; externally peer reviewed.

\section{REFERENCES}

1 Giuliano AR, Nyitray AG, Kreimer AR, et al. EUROGIN 2014 roadmap: differences in human papillomavirus infection natural history, transmission and human papillomavirus-related cancer incidence by gender and anatomic site of infection. Int J Cancer 2015;136:2752-60.

2 de Martel C, Ferlay J, Franceschi S, et al. Global burden of cancers attributable to infections in 2008: a review and synthetic analysis. Lancet Oncol 2012;13:607-15.

3 WHO. Globocan: Estimated Cancer Incidence, Mortality and Prevalence Worldwide. 2012.

4 Schiffman $\mathrm{M}$, Castle PE, Jeronimo J, et al. Human papillomavirus and cervical cancer. Lancet 2007:370:890-907.

5 Joura $E A$, Giuliano $A R$, Iversen $O E$, et al. A 9-valent HPV vaccine against infection and intraepithelial neoplasia in women. N Engl J Med 2015;372:711-23.
6 Katki HA, Kinney WK, Fetterman B, et al. Cervical cancer risk for women undergoing concurrent testing for human papillomavirus and cervical cytology: a population-based study in routine clinical practice. Lancet Oncol 2011;12:663-72.

7 Ronco G, Dillner J, Elfström KM, et al. Efficacy of HPV-based screening for prevention of invasive cervical cancer: follow-up of four European randomised controlled trials. Lancet 2014:383:524-32.

8 Ang KK, Harris J, Wheeler R, et al. Human papillomavirus and survival of patients with oropharyngeal cancer. N Engl J Med 2010;363:24-35.

9 Dok R, Nuyts S. HPV positive head and neck cancers: molecular pathogenesis and evolving treatment strategies. Cancers (Basel) 2016;8.

10 Beltrão M, Wanderley MS, de Santana NA, et al. Site of infections associated with human papillomavirus. Arch Gynecol Obstet 2015;291:481-91.

11 Kriegsmann $M$, Arens $N$, Endris $V$, et al. Detection of KRAS, NRAS and BRAF by mass spectrometry - a sensitive, reliable, fast and cost-effective technique. Diagn Pathol 2015;10:132

12 National Cancer Institute: Surveillance E, and End Results Program. SEER Stat Fact Sheets: Cervix Uteri Cancer. Secondary SEER Stat Fact Sheets: Cervix Uteri Cancer. http://seer.cancer.gov/statfacts/html/cervix.html

13 Munagala R, Donà MG, Rai SN, et al. Significance of multiple HPV infection in cervical cancer patients and its impact on treatment response. Int I Oncol 2009;34:263-71.

14 Patel DA, Shih YJ, Newton DW, et al. Development and evaluation of a PCR and mass spectroscopy (PCR-MS)-based method for quantitative, type-specific detection of human papillomavirus. J Virol Methods 2009;160:78-84.

15 Gravitt PE, Coutlee F, Iftner T, et al. New technologies in cervical cancer screening. Vaccine 2008;26(Suppl 10):K42-52.

16 Cooper WA, Yu B, Yip PY, et al. EGFR mutant-specific immunohistochemistry has high specificity and sensitivity for detecting targeted activating EGFR mutations in lung adenocarcinoma. J Clin Pathol 2013;66:744-8.

17 Cricca M, Marasco E, Alessandrini F, et al. High-throughput genotyping of high-risk Human Papillomavirus by MALDI-TOF Mass Spectrometry-based method. New Microbiol 2015;38:211-23.

18 Vaccarella S, Söderlund-Strand A, Franceschi S, et al. Patterns of human papillomavirus types in multiple infections: an analysis in women and men of the high throughput human papillomavirus monitoring study. PLOS ONE 2013;8: e71617.

19 Soderlund-Strand A, Dillner J. High-throughput monitoring of human papillomavirus type distribution. Cancer Epidemiol Biomarkers Prev 2013;22:242-50.

20 Jing L, Zhong X, Huang W, et al. HPV genotypes and associated cervical cytological abnormalities in women from the Pearl River Delta region of Guangdong province, China: a cross-sectional study. BMC Infect Dis 2014;14:388.

21 Yi X, Li J, Yu S, et al. A new PCR-based mass spectrometry system for high-risk HPV, part I: methods. Am J Clin Pathol 2011;136:913-19.

22 Peng J, Gao L, Guo J, et al. Type-specific detection of 30 oncogenic human papillomaviruses by genotyping both E6 and L1 genes. J Clin Microbiol 2013;51:402-8

23 Basu P, Chandna P, Bamezai RN, et al. MassARRAY spectrometry is more sensitive than PreTect HPV-Proofer and consensus PCR for type-specific detection of high-risk oncogenic human papillomavirus genotypes in cervical cancer. J Clin Microbiol 2011:49:3537-44. 